\title{
D2ER: Distributed Energy Efficient Routing in Wireless Sensor Networks with Unreliable Links
}

\author{
Dianjie Lu ${ }^{1,2}$, Xiangwei Zheng ${ }^{1,2}$, Guijuan Zhang ${ }^{1,2}$, Jue Hong ${ }^{3}$ \\ ${ }^{1}$ School of Information Science and Engineering, Shandong Normal University, Jinan, China \\ ${ }^{2}$ Shandong Provincial Key Laboratory for Novel Distributed Computer Software Technology, Jinan, China \\ ${ }^{3}$ Shenzhen Institutes of Advanced Technology, Chinese Academy of Sciences, Shenzhen, China \\ ludianjie@sina.com,sdnuzxw@126.com, guijuanzhang@gmail.com, honshj@gmail.com
}

\begin{abstract}
Wireless Sensor Networks (WSNs) are subject to node failures because of limited energy and link unreliability which makes the design of routing protocols in such networks a challenging task. The multipath routing scheme is an optimal alternative to address this problem which splits the traffic across multiple paths instead of routing all the traffic along a single path. However, using more paths introduces more contentions which degrade energy efficiency. The problem becomes even more difficult in the scenario of multiple source-destination pairs since the flow distribution of each link is uncertain. Our goal is to minimize the energy cost and provide the robust transmission by choosing the optimal paths. We propose a Distributed Energy Efficient Routing protocol (D2ER) to achieve this goal. In D2ER, we first study the problem from a theoretical standpoint by mapping it to the multi-commodity network design problem. Then, we employ the transportation method to solve this problem since it is hard to build a global addressing scheme due to the great number of sensor nodes. Simulation results demonstrate that our optimal algorithm can save energy drastically.

Index Terms - Energy efficient; wireless sensor networks; routing
\end{abstract}

\section{Introduction}

WSNs have been used for a wide range of applications such as military surveillance, disaster management and scientific exploration [1]. In WSNs, unpredictable nature of the wireless environment (e.g., channel fading or obstructions) usually leads to path failures and data loss. Empirical result from Berkeley [2] shows that the average packet delivery ratio reduces $5 \%-10 \%$ per link in sensor networks. Thus, in traditional single path routings, a source node prefers to select a node with high link quality as its own successor. As shown in Fig. 1, the source node S1 has two links to forward data to the destination D1. The weight of each link is the delivery ratio. Obviously, the source selects the link with higher delivery ratio for reliability. However, the single link makes the path delicate and the excessive energy cost of the nodes with high link quality drops the network lifetime. Recently, multipath routing approaches are widely utilized to address this problem. Different to single path routing methods, multi-path routing approaches split the traffic across multiple paths instead of routing all the traffic along a single path, which makes transmissions more resilient to failures. Moreover, the consumed energy spreads evenly across the nodes within the network, potentially resulting in longer lifetimes. However, the problem becomes difficult when multiple source nodes transmit simultaneously since the flow distribution among all the nodes is uncertain. For example, as shown in Fig. 2, source nodes S1 and S2 have the highest delivery ratio on links to the same relay node 2 . To avoid the congestion on node 2 , both source nodes should transmit part of packets on the links to node 1 and node 3 respectively. In addition, sensor nodes power is supplied by batteries which are subject to a critical constraint for different applications. Using multipath to sending packets is proved inducing more energy cost since more paths introduces more contentions [9]. Therefore, how to design energy efficient multipath routing protocols to satisfy the performance requirements of multiple source-destination pairs is a crucial issue.

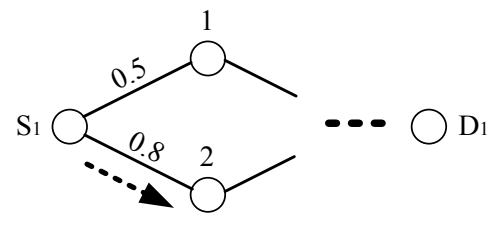

Fig. 1 The single path

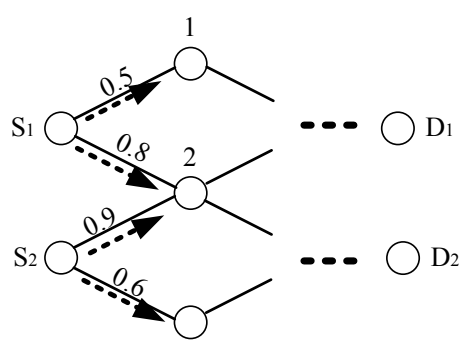

Fig. 2 The multi-path

In this paper, we consider both reliability and energy constraints in multipath routing. Here, reliability is defined as the packet delivery ratio. Our goal is to find ways for energy efficient route setup and reliable relaying of data from the

\footnotetext{
* This work is supported by National Natural Science Foundation of P.R.China under Grant Nos. 61202225, 61272094, 61202417, and Shenzhen Basic Research Foundation under Grant No. JC201105190934A.
} 
sensor nodes to the sink so that the lifetime of the network is maximized.

The rest of the paper is organized as follows. Section 2 provides a brief overview of existing multipath routing approaches. Section 3 presents our system model. In Section 4, we give the distributed energy efficient routing algorithm. Then, we evaluate the performance of our proposed approach in Section 5. Finally, we conclude the paper in Section 6.

\section{Related Work}

Energy efficient routing has always been a hot research topic in wireless networks [3], [4]. Some researchers propose energy aware routing schemes by using the residual battery power as a routing metric [5]-[7]. In [5], the scheme routes data through a path whose nodes have the largest residual energy. An established path is used until a path with more residual energy is discovered. Thus, nodes will not completely exhaust their energy by using different paths, so that the lifetime of the whole system may be prolonged. However, they assume that each link is totally reliable without considering retransmission when computing energy efficient paths. To achieve better energy efficiency in realistic scenarios, the metrics with the capability of handling packet losses in the wireless environment should be considered. Banerjee et. al. [6] explores the impact of unreliable links on energy efficient routing and solves the problem of finding minimum energy paths hop-by-hop. In this approach, each node retransmits lost packets as and when necessary. In [7], the authors solve the problem of computing minimum energy paths for reliable transmission in the end-to-end retransmission model where none of the links guarantees any reliability. However, these works mentioned above only consider the scenario of one source-destination pair.

A multipath routing scheme that finds several disjoint paths is presented in [8]. This protocol tends to improve the reliability to node failures. In their work, there are two kinds of multipath schemes are proposed: the classical node disjoint multipath, and a braided multipath that consists of partially disjoint alternate paths. The objective using these braided paths is to keep the overhead of maintaining the multiple paths low. In this proposal, source nodes or intermediate nodes select one path from the available multiple paths to transmit the data to sink based on the quality of links (e.g. delay, throughput). By observing the variations on the network conditions, a node may change its primary path to another one. However, this scheme leads to a drastically increasing energy cost because of frequently switching to or establishing a new primary path in case of unreliable network environments. In this paper, we design an energy efficient multipath routing protocol in a multiple source-destination scenario considering the factor of energy consumption.

\section{System Model}

\section{A. Network Model}

We consider an ad hoc sensor network consisting of a set of $\mathrm{N}$ nodes. We consider a set of $\mathrm{L}$ concurrent multi-hop sessions. Each session $\ell \in \mathrm{L}$ corresponds to a flow $f$ with a source-destination pair $(s(\ell), d(\ell))$ in the network. The traffic demand for each session $\ell$ is given by $W(\ell)$ (bits). Traffic of a particular session may be split to sub-flows routed over different paths. The choice of these routing paths depends on the underlying schedule of different concurrent transmissions. The objective of our model is to minimize the energy consumption for delivering the $\mathrm{L}$ sessions without violating the interference constraints.

\section{B. Expected Transmission Count}

In this paper, we investigate the delivery ratio by the Expected Transmission Count (ETX) [9]. ETX is calculated with the forward and reverse delivery ratio of the link. The forward delivery ratio $d_{f}$ is the measured probability that a data packet successfully arrives at the recipient. Likewise, the reverse delivery ratio $d_{t}$ refers to the probability that the ACK packet is successfully received. Thus, the probability that a packet is successfully received and acknowledged is $d_{f} \cdot d_{r}$. A sender will retransmit a packet that is not successfully acknowledged. Because each transmission attempt can be considered as a Bernoulli trial, ETX can be written as

$$
\eta=\frac{1}{d_{f} \times d_{r}} .
$$

The delivery ratios $d_{f}$ and $d_{r}$ can be measured using probe packets. First, we broadcast probes of fixed size in every period. Then, we count the successfully received probes at the recipient node. $d_{f}$ can be derived from the following formula

$$
d_{f}=\frac{\operatorname{count}(t-w, t)}{w / \tau},
$$

where count $(t-W, t)$ is the number of probes received during window $W, W / \tau$ is the number of probes that should be received. Similarly, the reverse delivery ratio $d_{r}$ can be computed at the sender.

\section{Distributed Energy Efficient Routing}

The objective of our model is to minimize the system energy consumption for delivering the $\mathrm{L}$ sessions. In the distributed routing scheme, a path can be established hop by hop which saves a large amount of computation since per hop information is convenient to acquire. Similarly, we also consider the flow transmission problem hop by hop. At each hop, we employ the transportation method to optimize the flow distribution with minimal energy consumption.

\section{A. Formulation}

We assume each link corresponds to a start-target pair. The traffic demand for each link is given by $f_{i j}$ (bits). Traffic of a particular session may be split to sub-flows routed over different paths. The choice of these routing paths depends on the underlying schedule of different concurrent transmissions. 
Mathematically, to transfer the traffic from starts to their targets with minimum cost, we consider the two nodes sets: the start set $U_{s}=\left\{u_{s}^{1}, \cdots, u_{s}^{m}\right\}$ and the target set $U_{t}=\left\{u_{t}^{1}, \cdots, u_{t}^{n}\right\} \cdot u_{s}^{i}(1 \leq i \leq m)$ and $u_{t}^{j}(1 \leq j \leq n)$ are node representatives, where $m$ is the number of start nodes and $n$ is the number of target nodes in a single hop.

We denote $v\left(u_{s}^{i}\right)$ as the demand-traffic of $u_{s}^{i}$, and $v\left(u_{t}^{j}\right)$ as the relay-traffic of $u_{t}^{j}$ which measures the relay capability of an intermediate node. Let $P=\left\{\left(u_{s}^{1}, v\left(u_{s}^{1}\right)\right), \cdots,\left(u_{s}^{m}, v\left(u_{s}^{m}\right)\right)\right\}$ be the state representation of $m$ start nodes and $Q=\left\{\left(u_{t}^{1}, v\left(u_{t}^{1}\right)\right), \cdots,\left(u_{t}^{n}, v\left(u_{t}^{n}\right)\right\}\right.$ be the state representation of $n$ target nodes. So, the problem of transferring the traffic from start nodes to their target nodes is converted into the link matching problem which can be formulated as

$$
Q=F(P)
$$

The mapping $F=\left[f_{i j}\right]$ can transform $P$ into $Q$ (as shown in Fig.3). Thus, our further work is to find an optimal mapping where all the start nodes in $P$ can find the target nodes in $Q$ so that the total traffic can be transferred with minimum energy consumption.

We denote $D=\left[\eta_{i j}\right]$ as the expected transmit count matrix among nodes in $U_{s}$ and $U_{t}$. Here, the expected transmit count between two nodes $u_{s}^{i}$ and $u_{t}^{j}$ is $\eta_{i j}=1 /\left(d_{f_{i j}} \times d_{r_{i j}}\right)$. In this paper, we assume a unit energy cost, denoted $\xi$, equals to the energy consumption of transmitting a bit. The entry $f_{i j}$ of $F$ denotes the traffic needed to be transported from $u_{s}^{i}$ to $u_{t}^{j}$. So, the optimization problem which minimizes the energy consumption can be expressed as

$$
\operatorname{Cost}(P, Q, D, F)=\operatorname{Minimize}\left(\sum_{i \in U_{s}} \sum_{j \in U_{t}} f_{i j} \eta_{i j} \xi\right)
$$

Subject to :

$$
\begin{array}{lr}
f_{i j} \geq 0 & 1 \leq i \leq m, 1 \leq j \leq n \\
\sum_{j \in U_{t}} f_{i j}<v\left(u_{s}^{i}\right) & 1 \leq i \leq m \\
\sum_{i \in U_{s}} f_{i j}<v\left(u_{t}^{j}\right) & 1 \leq j \leq n \\
\sum_{i \in U_{s}} \sum_{j \in U_{t}} f_{i j}=\min \left(\sum_{i \in U_{s}} v\left(u_{s}^{i}\right), \sum_{j \in U_{t}} v\left(u_{t}^{j}\right)\right) .
\end{array}
$$

Equation (4) shows an objective function followed by a set of constraints. As for the first constraint, Equation (5) means that the traffic transported between two nodes $u_{s}^{i}$ and $u_{s}^{i}$ is no less than 0. Equation (6) denotes that the traffic shipping from a start node is no more than its demand-traffic. Similar situation exists in Equation (7). That means the traffic received from start nodes is no more than the relay-traffic of a target node. As for Equation (8), the total shipping traffic from start nodes to target nodes equals to the smaller one of total demand-traffic and total relay-traffic.

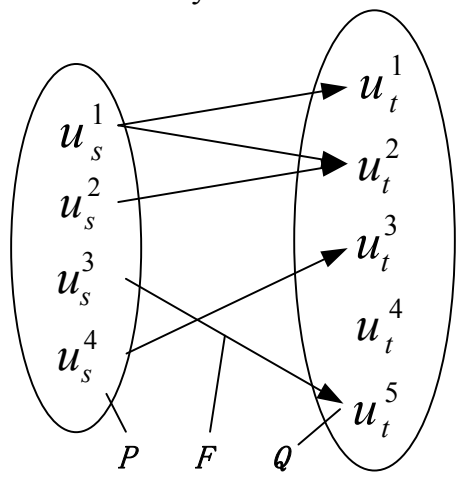

Fig. 3 The Mapping F between P and Q.

\section{B. Matching solution}

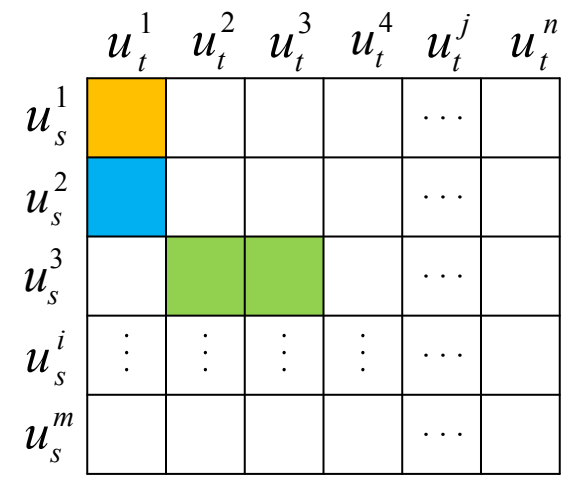

Fig. 4 The solution to our transportation problem: flow matrix. It records the matching result and the traffic load that transported among start and target nodes. The colourful elements denote values greater than 0 . In the third row, $\mathrm{u}_{\mathrm{s}}{ }^{3}$ matches with $\mathrm{u}_{\mathrm{t}}{ }^{2}$ and $\mathrm{u}_{\mathrm{t}}{ }^{3}$. In the first column, two start nodes $\mathrm{u}_{\mathrm{s}}{ }^{1}$ and $\mathrm{u}_{\mathrm{s}}{ }^{2}$ are matched with $u_{t}{ }^{1}$. It means that these two nodes have the same target node $u_{t}{ }^{1}$.

In this part, we employ a well-known transportation problem [10] to find the optimal matching result between each start node and target node. The solution of the transportation problem is a flow matrix which determines the minimum energy consumption required to transport packets to the target.

Given $P, Q$ and $D$, we use the efficient transportation method [10] to obtain the matching result among the start nodes and the target nodes by solving the transportation problem. It includes two essential steps: 1) determine the initial basic feasible solution; 2) test for optimization using the stepping stone or MODI method [11]. In the first step, the Vogel's approximation [12] is adopted since it gives an initial basic feasible solution which is much closer to the optimal solution. It reduces the total number of iterations required in the second step to reach an optimal solution considerably. Details of solving the transportation problem can be referred to literature [10]. 
The solution is denoted as a flow matrix $F=\left[f_{i j}\right]$. Any nonzero entry $\left(f_{i j}>0\right)$ implies that $u_{s}^{i}$ matches with $u_{t}^{j}$, and the shipping traffic between $u_{s}^{i}$ and $u_{t}^{j}$ equals to $f_{i j}$. Fig.4 gives an example of the flow matrix. In the example, the flow matrix records the matching result and the traffic load that transported among start and target nodes. The colourful elements denote values greater than 0 . In the third row, $u_{s}^{3}$ matches with $u_{t}^{2}$ and $u_{t}^{3}$. In the first column, two start nodes $u_{s}^{1}$ and $u_{s}^{2}$ are matched with $u_{t}^{1}$. It means that these two nodes have the same target node $u_{t}^{1}$.

The transportation method is guaranteed to converge. Transportation problem is a special type of linear programming, and it is proved that the problem has an optimal solution. Also, transportation method belongs to the simplex method. In the literature [11], Dantzig demonstrated that the simplex method can find the optimal solution (if exists) in a finite number of iterations. Therefore, our method can find the optimal solution and converges in limited iteration steps.

\section{Performance Evaluation}

In this section, we investigate the energy consumption of a single-hop scenario in terms of number of start nodes and the traffic load of start nodes.

\section{A. Number of Start Nodes}

We assume that the traffic loads of start nodes and target nodes are both uniformly distributed over [1 10] Mbps. We set the number of target nodes with a fixed value which equals to 4 and show the improvement of the proposed algorithm with respect to the number of start nodes. It can be seen from Fig. 5 that as the number of start nodes rises, the energy consumption is greatly increased. We also see that our optimal algorithm outperforms the greedy and the random algorithms and reduces the energy consumption by up to $28 \%$ and $35 \%$ as the number of start nodes rises.

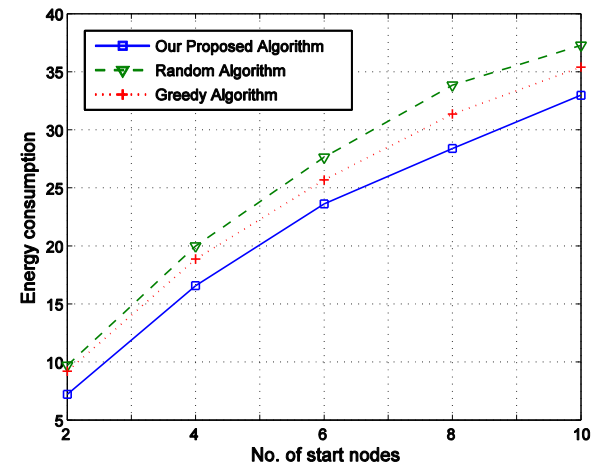

Fig. 5 Energy consumption vs. Number of start nodes

\section{B. Average Throughput}

We compare the energy consumption of three approaches with respect to the traffic load of start nodes. We set the number of start nodes and target nodes with fixed value which equals to 6 and 4 respectively. Fig. 6 demonstrates that the energy consumption is greatly increased as the traffic load of start nodes rises. It is shown that the energy consumption of our optimal approach is lower than that of the greedy and the random algorithms by up to $16 \%$ and $24 \%$ respectively.

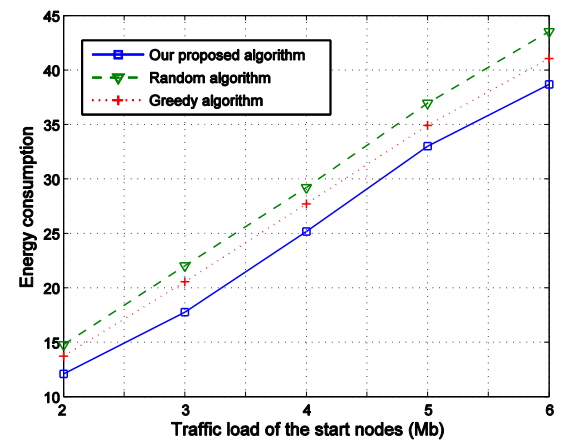

Fig. 6 Energy consumption vs. Traffic load of start nodes

\section{Conclusion}

In WSNs, multipath routing strategies have been proposed to improve the performance of reliability and energy balance. However, using multipath to sending packets leads to more energy cost since more paths introduces more contentions. In this paper, we consider both reliability and energy constraints in multiple source-destination pairs scenario to find the optimal energy efficient and reliable relaying of data from the sensor nodes to the sink so that the energy cost is saved drastically. Simulation results show the effectiveness of our approach.

\section{References}

[1] J. Yick, B. Mukherjee, and D. Ghosal. Wireless Sensor Network Survey, Computer Net-works, vol. 52, pp. 2292-2330, 2008

[2] N. Ota, D. Hooks, P. Wright, D. Ausiander and T. Peffer. Poster abstract: wireless sensor networks characterization-application to demand response energy pricing, In Proc. of ACM SenSys'03, pp. 334-335, Nov. 2003.

[3] S. K. Singh, M. P. Singh, D. K. Singh. A Survey of Energy-Efficient Hierarchical Cluster-Based Routing in Wireless Sensor Networks, Journal of Advanced Networking and Applications, vol. 02, pp. 570-580, 2010.

[4] B. Yahya and J. Ben-Othman. REER:Robust and Energy Efficient Multipath Routing Proto-col for Wireless Sensor Networks, In Proc. of GLOBECOM'09, pp. 1-7, Dec. 2009

[5] J. Chang and L. Tassiulas. Energy Conserving Routing in Wireless Adhoc Networks, In Proc. of INFOCOM'00, pp. 22-31, Mar. 2000.

[6] S. Banerjee and A. Misra. Minimum Energy Paths for Reliable Communication in Multi-hop Wireless Networks, In Proc. of ACM MobiHoc'02, pp. 146-156, June, 2002.

[7] Q. F. Dong, S. Banerjee, M. Adler and A. Misra. Minimum Energy Reliable Paths Using Un-reliable Wireless Links, In Proc. of ACM MobiHoc'05, May, 2005.

[8] D. Ganesan, R. Govindan, S. Shenker, and D. Estrin. Highly-resilient, Eenergy-efficient Multipath Routing in Wireless Sensor Networks, In Proc. of ACM SIGMOBILE'01, pp. 11-25, 2001.

[9] R. Draves, J. Padhye, and B. Zill. Routing in Multi-radio, Multi-hop Wireless Mesh Net-works, in Proc. of MobiCom'04, pp. 114-128, Sep. 2004.

[10] F. S. Hillier and G. J. Lieberman. Introduction to Operations Research,McGraw-Hill Sci-ence/Engineering/Math (2005).

[11] Dantzig and B. George. Linear Programming and Extensions, NJ: Princeton University Press(1963).

[12] N. V. Reinfeld and W. R. Vogel. Mathematical Programming, Prentice Hall, Englewood Cliffs, New Jersey (1958). 